\title{
Design of Indoor Intelligent Home System Based on Andrews and ZigBee Technology
}

\author{
ChenHong ${ }^{1, a^{*}}$ \\ ${ }^{1}$ School of computer science, Wuhan Donghu University, Wuhan,HuBei,430212 China \\ a30315495@qq.com
}

Keywords: Intelligent home; ZigBee; remote control; smart home appliances; father and son relationship

\begin{abstract}
The ZigBee wireless networking technology has the advantages of no wiring, flexible network, easy installation and expansibility, and the ZigBee communication module has low cost in the application of intelligent home system. The indoor intelligent Home system design system based on the Android and ZigBee Technology realizes the intelligent control of the switch type and the infrared type home appliance, and has certain development foreground.
\end{abstract}

\section{Introduction}

With the development of short-range wireless communication technology, ZigBee technology has been gradually applied to intelligent home, industrial control and environmental monitoring in many fields, so that people can directly control home appliances through the voice, can let people enjoy the modern technology in real life applications.

\section{Network analysis of indoor intelligent Home system design based on ZigBee technology}

Network analysis of indoor intelligent Home system design of ZIGBEE technology in ZigBee network, according to function can be divided into fully functional equipment and simplified functional equipment, according to the role is divided into routers, coordinators and terminals, routers are responsible for routing discovery and message forwarding, terminal devices only send and receive data. The ZigBee network has 3 kinds of network topology, such as tree, star and mesh network, and this paper is analyzed on the basis of mesh network.

\section{A. ZigBee Address Assignment}

Each node in ZigBee has 2 addresses, 64-bit IEEE extended addresses and 16-bit network addresses. Allocation mechanism allocates: Suppose $\mathrm{C} \mathrm{m}$ is the maximum number of child nodes that a parent node can hold, $\mathrm{R}_{\mathrm{m}}$ is the number of nodes in the corresponding child node, $\mathrm{L}_{\mathrm{m}}$ represents the maximum depth of the network, $d$ represents the network depth of the parent node, and the offset of the assigned address space $\mathrm{C}_{\text {skip }}(\mathrm{d})$ :

$$
\mathrm{C}_{\text {skip }}(\mathrm{d})=\left\{\begin{array}{c}
1+\mathrm{C}_{\mathrm{m}}\left(\mathrm{L}_{\mathrm{m}}-\mathrm{d}-1\right), \mathrm{R}_{\mathrm{m}}=1 \\
\frac{1+\mathrm{C}_{\mathrm{m}}-\mathrm{R}_{\mathrm{m}}-\mathrm{C}_{\mathrm{m}} \mathrm{R}_{\mathrm{m}}^{\mathrm{Lm}-\mathrm{d}-1}}{1-R_{m}} \text {, others }
\end{array}\right.
$$

The parent node address with the depth of $D$ is $A_{\text {parent }}$, and the address of its child node is $A_{n}$, if the address is assigned to several child devices with routing capabilities:

$$
\mathrm{A}_{\mathrm{n}}=\mathrm{A}_{\text {paren }} \mathrm{t}+1+\mathrm{C}_{\text {skip }}(\mathrm{d}) \times(\mathrm{n}-1) 1 \leq \mathrm{n} \leq \mathrm{C}_{\mathrm{m}}-\mathrm{R}_{\mathrm{m}}
$$

The address assigned to a terminal device can be performed as a formula (3), starting with the last router:

$$
A_{n}=A_{\text {parent }}+1+C_{\text {skip }}(d) \times R_{m}+n \quad 1 \leq n \leq C_{m}
$$

\section{B. ZigBee Routing algorithm}

ZigBee supports Cluster-tree, AODVJR, and CLUSTER-TREE+AODVJR, and in most cases the 3rd algorithm is used for fewer mobile and stationary situations so that routing tables are not stored. AODVJR is based on an on-demand distance vector routing protocol to simplify the 
Protocol. AODV routing protocol provides a method for routing in mobile Adhoc networks, and AODV has many unique functions: establishing mobile networks, establishing routing paths on demand, good scalability, and conforming to existing protocol stack specifications. In the route discovery process, the routing table of the node is updated according to the path cost, and the path with the lowest path cost is selected. For a RN1 node of a router, if the data is forwarded to the destination node and its address is D, the node address of the known router is A, the depth is d, and the formula (4) obtains whether the destination is a child node:

$$
\mathrm{A}<\mathrm{D}<\mathrm{A}+\mathrm{C}_{\text {skip }}(\mathrm{d}-1) \quad \text { (4) }
$$

To satisfy the above, we can determine the RN1 of the router. The parent node determines the process as shown in Figure 1. Start with the coordinator of address 0, calculate to the next hop address $\mathrm{N}$ of the router, when calculating a node's address is $\mathrm{N}=\mathrm{D}$, it can determine that this destination node is RN1 parent node, instead of RN1 parent node.

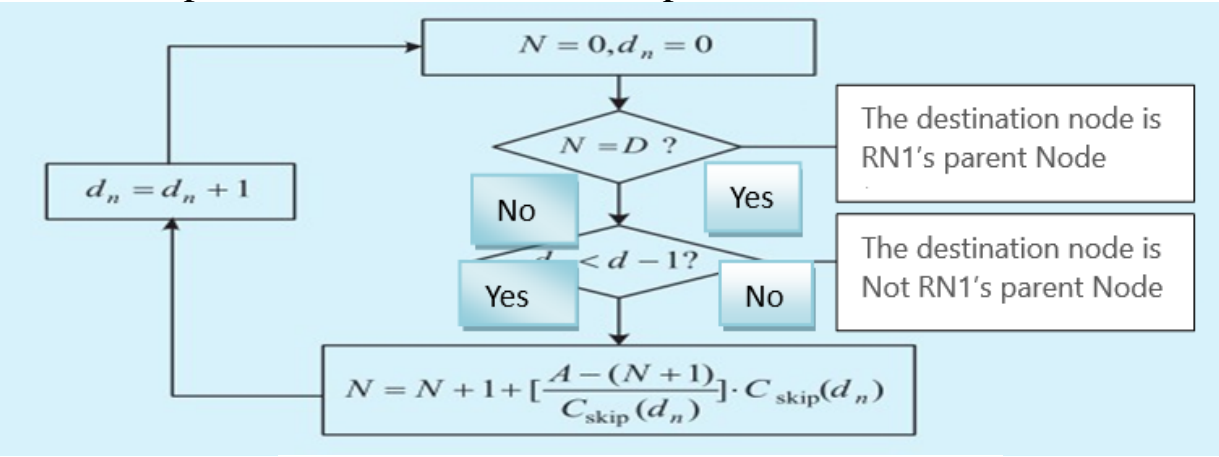

Figure 1 The parent node judgment process

\section{Android Project Organizational Structure}

A standard Android application typically consists of four parts, the activity, broadcast Receiver, Service, and Content Provider, which are also known as the four components of Android. Activity: Each individual interface of an application's display, in which the UI interface can be drawn, and the lifecycle of the activity is shown in Figure 2:

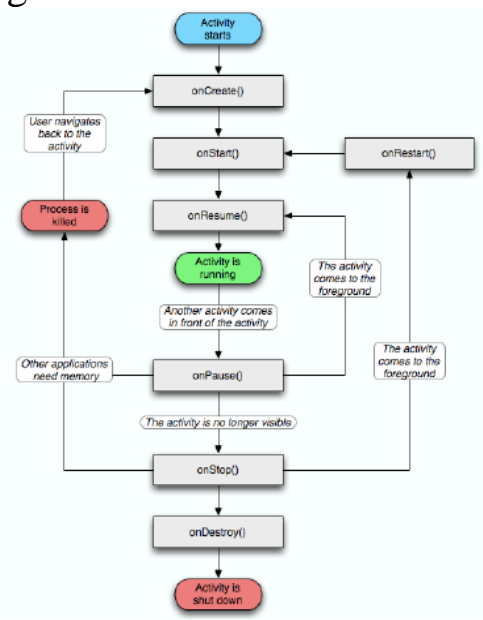

Fig. 2 life cycle of the activity

Broadcastreceiver: Broadcast receivers, receiving and processing through broadcastreceiver, developers can also customize broadcast events, as long as registering it in the system AndoirdManifest.xml. Service: Service is a long lifecycle that Android provides, and is typically used to handle monitoring and time-consuming operations. Remote services can be applied between different applications within the Andoird system for operation by other applications. ContentProvider: Content provider. This component is used primarily for data preservation and sharing. It can provide a unified interface for storing and reading data, using ContentProvider, sharing data between different applications, and Andoird built-in many of the data to be called by the developer in contentprovider form. These four components have taken a particularly important 
role in the development of this system. The activity is responsible for drawing the interface and responding to user events. Broadcast Receiver in this system mainly listens to the telephone incoming event. Service in this system is mainly used to monitor the long connection Socket operation. Content Provider in this system mainly through the unified interface call SQLite database, used to save and read data.

\section{Design of Indoor intelligent home system based on Android and ZigBee technology}

The data the Android phone sends to the Zigbee coordinator is mainly responsible for querying the environment information of single or multiple Zigbee nodes, querying the relevant basic information of the node and setting the threshold value of the alarm. In order to meet the above features, the packet format is shown in table 1.

Table 1 Android phone to ZigBee Coordinator packet

\begin{tabular}{|l|l|l|l|l|}
\hline Type of action & Action Content & Node address & Node type & Reserved fields \\
\hline
\end{tabular}

Operation type: This field occupies 1 bytes, 0x01 means querying a single node information, 0x02 to query all node information and 0x03 to set alarm threshold function.

Table 2 Conventions for manipulating type fields

\begin{tabular}{|c|c|}
\hline HEX value & Action type \\
\hline 0x01 & $\begin{array}{l}\text { Querying a single ZigBee node } \\
\text { information }\end{array}$ \\
\hline $0 \mathrm{x} 02$ & $\begin{array}{c}\text { Querying all ZigBee } \\
\text { information }\end{array}$ \\
\hline $0 \times 03$ & Setting thresholds \\
\hline
\end{tabular}

Operation content: This field occupies 1 bytes, 0x01 represents query environment data information function, 0X02 represents the basic information of the node, 0x03 represents the value of the specific alarm threshold value.

Table 3 Conventions for manipulating content fields

\begin{tabular}{|l|l|}
\hline HEX value & Action type \\
\hline $0 \times 01$ & Environmental data \\
\hline $0 \times 02$ & Node information \\
\hline $0 \times 03$ & Alarm thresholds \\
\hline
\end{tabular}

Node type: This field occupies one byte, that is, the specific type of query data in the Zlgbee node. 0X01 indicates that the query is temperature and humidity data, 0x02 indicates the time brightness data of the query, 0x03 indicates that the query is infrared data information, 0x04 indicates that the query is smoke data information.

Table 4 Conventions for table node type fields

\begin{tabular}{|l|l|}
\hline HEX value & Action content \\
\hline $0 \times 01$ & Temperature and humidity \\
\hline $0 \times 02$ & Light brightness \\
\hline $0 \times 03$ & Infrared \\
\hline $0 \times 04$ & Smoke \\
\hline
\end{tabular}

Use MMS to deliver multimedia information: The first step is to build the MMS content that you send as long as you create a PDU object, which requires MMS from the Andoird source MMS application. Copy all of the implementation classes from the pdu package into the project. By calling the following code, you can construct a brilliant letter PDU object, which is a pduBody variable in this project.

PduBody pduBody=new PduBody();

PduPart part=new PduPart();

part .setName("sample".getBytes())

part .setContentType("image/jpeg” getBytes()); 
PduPart partPdu= new PduPart( );

partPdu.setCharset(CharacterSets. UTF_8)

partPdu.setName(part.getName());

partPdu.setContentType(part.getContentType());

partPdu.setDataUri(Uri.parse(furl));

pduBody .addPart(partPdu);

sendReauest .setBody(pduBody);

The second step is to send the pdu object assembled in the first step to the MMS center. Different card pairs for mobile, Unicom and Telecom

The MMS Center address is not the same. You can refer to the following,

Related properties of China Mobile MMS Center:

URL:http://mmsc.monternet.com

Proxy:10.0.0.172

Port:80

China Telecom MMS Center related properties:

URL:http://mmsc.vnet.mobi

Proxy:10.0.0.200

Port:80

China Unicom MMS Center related properties:

URL:http://mmsc.myuni.com.cn

Proxy:10.0.0.172

Port: 80

Call the code snippet and send it to the corresponding MMS center.

The silent sending of MMS under the Android system is not related to the api, the main process of image monitoring is as follows: The first step, outdoor Android send the SMS instructions monitored by pictures to the indoor Android phone. The second step, the indoor Android phone received SMS instructions and to resolve the instructions. In the third step, the indoor Android phone calls the custom camera module to shoot the surroundings. Step fourth, the indoor Android phone locally to save the pictures. In step fifth, the indoor Android phone will send the image from step fourth to an outdoor Android phone via an email module. Step sixth the outdoor Android phone receives an e-mail with picture information to view. Complete the function of picture monitoring.

\section{Conclusion}

With the improvement of people's living standards, people have a higher requirement for the safety and reliability of their living environment, and they are eager to install a smart home monitoring system in their home. In this paper, a scheme of using Android and ZigBee technology to build a family monitoring system is proposed.

\section{Acknowledgements}

This paper is supported by Youth Foundation of Wuhan Donghu University.

\section{References}

[1] Zhu Yunle. Design and realization of intelligent home system[J]. Electronic quality, 2017, (08): 35-39.

[2] Yang Ling, Chen Weikang, Cheng Yong, Zheng Zhongren. Design and implementation of intelligent home system based on Android [J]. Information technology, 2017, (07): 19-22+25.

[3] Wang Ruina. Design and implementation of intelligent home system based on Android [J]. Journal of Hebei North College (Natural Science Edition), 2016, 32 (11): 8-13. 
[4] Wanghaitao, Hu Yuanlu. Design and implementation of intelligent home system for power carrier communication based on OFDM[J]. Communication power technology, 2017, 34 (03): 127-128.

[5]Li Hongri. Design and implementation of intelligent home system based on Android [J]. Computer knowledge and technology, 2017, 13 (03): 234-235. 